\title{
Numerical Analysis and Position Strategies of Tug-of-war
}

\author{
Yizhou TANG ${ }^{1}$, Xin $\operatorname{LIN}^{1, a,{ }^{*}}$ \\ ${ }^{1}$ School of Economics and Management, Tongji University, Shanghai, China \\ atyzlunwen@163.com \\ ${ }^{*}$ Corresponding author
}

Keywords: tug-of-war, position strategies, numerical analysis, simulation.

\begin{abstract}
This paper analyzes the mechanism of tug-of-war and establishes a numerical framework of it. Base on this framework, the position strategies problem is discussed. This paper makes a simulation and obtains two basic position strategies: "standing from high to low" and "the heavier you are, the further you stand". Lastly, this paper evaluates the current rules of distance for win. The simulation results show that the 4-meters to win rule is appropriate.
\end{abstract}

\section{Introduction}

Tug-of-war is a kind of amateur sports activity which is favored by almost everybody. Tug-of-war is a sport that directly puts two or more teams against each other in a test of strength: teams pull on opposite ends of a rope, with the goal being to bring the rope a certain distance in one direction against the force of the opposing team's pull. It can exercise its participants' muscles, legs, waist force and endurance. Also, it calls for team spirit of cooperation and is full of excitement. Once upon a time, tug-of-war was very popular around world. It was part of summer Olympic Games’ program during 1900-1920. However, there is little research and analysis on tug-of-war. People didn't know too much about the skills or strategies of this kind of sport.

In order to figure out the mechanism of tug-of-war and its related strategies and rules, this paper analyses the numerical model of it. Based on the reasonable assumptions we made, we simulate our model to study how the backward angle may influence the game and how much will the weight, height affect the game. Considering the differences of participants' weight and height, we analyze and carry out the best position strategy for tug-of-war. At last, we discuss the appropriate pulling distance for win. By assuming different weight class team subject to different pulling force, we simulate those situations. According to the simulation results, we then check the validity of the current rules about winning distance.

The rest of the paper is organized as follows. The next section analyses the numerical model and its simulation. The position strategies are also discussed in this section. Section 3 evaluates the rules' validity. Conclusion is given in section 4 .

\section{Numerical model and simulation}

\section{Basic assumptions and symbols description.}

Before establishing our models, we make the following assumptions:

1) The rope in our analysis is very light, and the mass of it can be ignored.

2) The competition is held between two groups of participants and their total weight is similar.

3) In our model, the human body is seemed as rigid body.

4) During the game, there is no sliding between the rope and the participant's hands.

5) The differences between participants are limit to power, height and weight.

Model Analysis.

To make force analysis, we seem the whole team as one man with his height is $h$. The figure 1 shows the force analysis. 


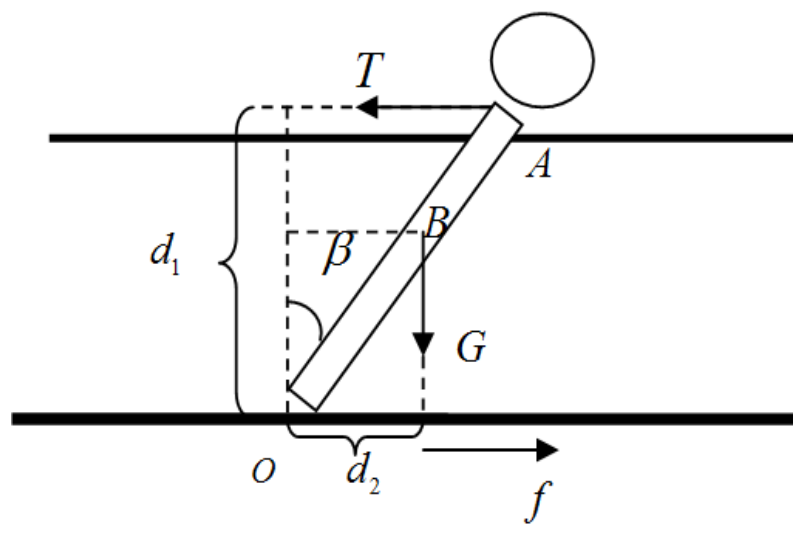

Figure 1. Force analysis of man

In steady state, we have frictional force $f=\mu \cdot m \cdot g, f=T$ and both teams are stay in a balance. As for moment of force, the moment of gravity $M_{1}=G \cdot d_{2}$, the moment of pulling force $M_{2}=T \cdot d_{1}$. When moments are balance, we have $M_{1}=M_{2}$. The resultant moment $M=0$. Then we assume the center of gravity of human body is at its middle point B. So we can obtain the moment arm of force $d_{1}, d_{2}$.

$$
\begin{aligned}
& d_{1}=h \cdot \cos \beta \\
& d_{2}=\frac{1}{2} h \cdot \sin \beta
\end{aligned}
$$

Then we have:

$$
\begin{aligned}
& f=T \\
& T \cdot d_{1}=G \cdot d_{2}
\end{aligned}
$$

There are two situations will produce a winner. According to Newton's Third Law, applied force and reactive force have the same magnitude. When one team's frictional force is smaller than the pulling force from the rope, which will produce translational motion. If the frictional force is equal with the puling force, we should analyze the moment. When one team's moment of gravity is smaller than the moment of pulling force, rotation will occur. If the force and moment are all in balance, the more endurance team will break the balance first.

In a tug-of-war game, as we can see, participants are always lean backward. The bigger angle you lean, the moment of gravity will be larger and the moment of pulling force will be smaller. However, the angle can be too big because the increment of pulling force should not exceed the frictional force:

$$
T=m \cdot g \frac{d_{2}}{d_{1}} \leq \mu \cdot m \cdot g
$$

Then we obtain:

$$
\mu=\frac{1}{2} \tan \beta
$$

Thus we can know that $\beta \leq \arctan 2 \mu$. The $\beta$ here is called frictional angle. If the backward angle is smaller than fractional angle, there will be no sliding. This phenomenon is called "self-locking”. Accordingly, we know that the coefficients of friction of cement court and rubber floor are range from 0.6 to 0.85 . We take $\mu=0.8$ into calculation and figure out that the biggest backward angle is limit to 58 degree. 


\section{Position Strategy.}

Based on the basic model established above, we analyze the position strategy. The differences between participants are limit to power, height and weight.

Analysis of the body height is shown in the figure 2. If a team is at a high level, pulling force forms an angle with the horizon. It may produce a downward force to the higher one and bigger frictional force respectively. Absolutely the higher one has more advantages.

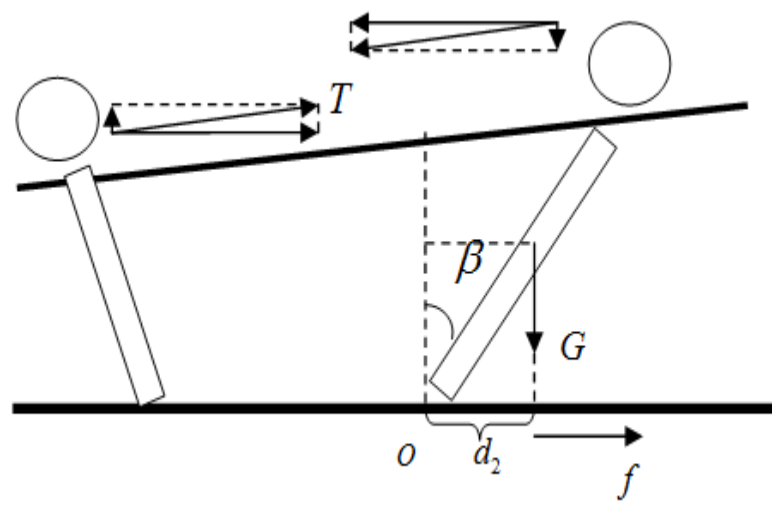

Figure 2. Analysis of the body height

Then we seem the teammates as a whole, which like a brick.

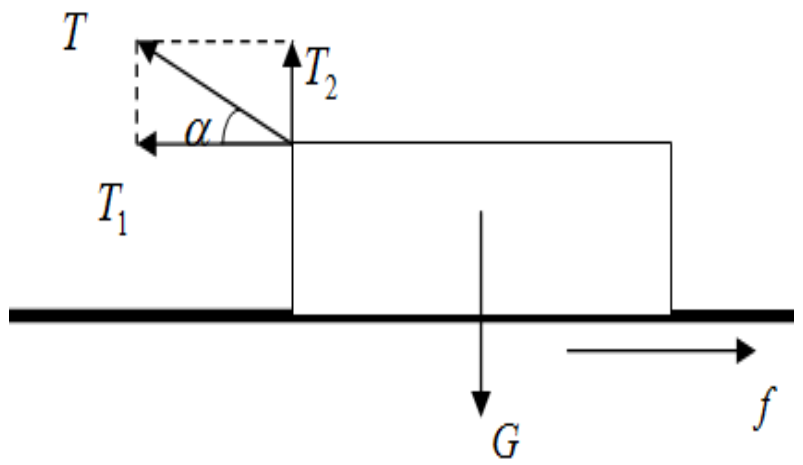

Here we have:

Figure 3. Global analysis

$$
\begin{aligned}
& f=\mu(G-\sin \alpha T) \\
& T_{1}=\cos \alpha \cdot T
\end{aligned}
$$

We assume the distance between two teams is 8 meters and the height gap is 0.2 meters. So the angle $\alpha=\arctan 0.2 / 8 \approx 1.4^{\circ}$. Then we use MATLAB to simulate this situation.

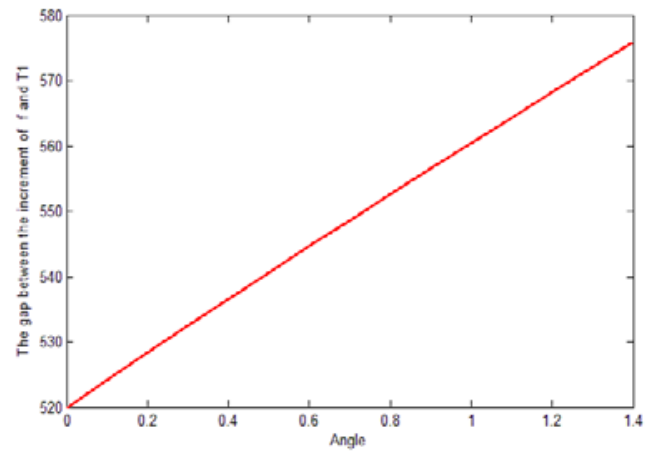

Figure 4. Analysis of the angle’s influence 
As we can see from the figure 4, if there is a 0.2 meters gap between two teams, the pulling force gap from the rope will be 60 newtons, which may even determine the game results.

Then we compare two kinds of position strategies: standing from high to low and standing from low to high. The force analyses of these two strategies are as figure 5.

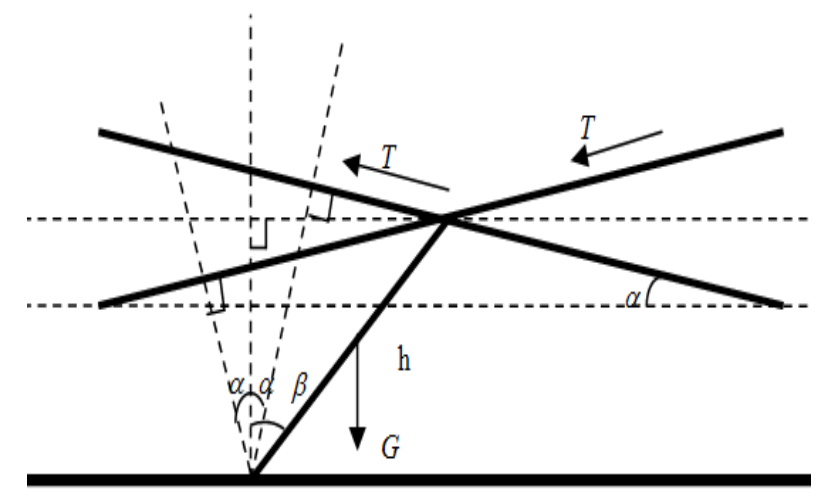

Figure 5. Analysis of the strategies

We assume force and moment are all in balance. According to equation (3) and (4), we can deduce:

$$
T=m g \frac{1}{2} \tan \beta
$$

The distance between two teams is still assumed as 8 meters and the height gap is 0.2 meters. We simulate the two strategies' impact. We can see from the Figure 6, when team stand from high to low, the maximum pulling force is increase with bigger angle. It indicates this kind of strategy can stand bigger moment of pulling force. The bigger moment you can stand, the harder you are dragged away.

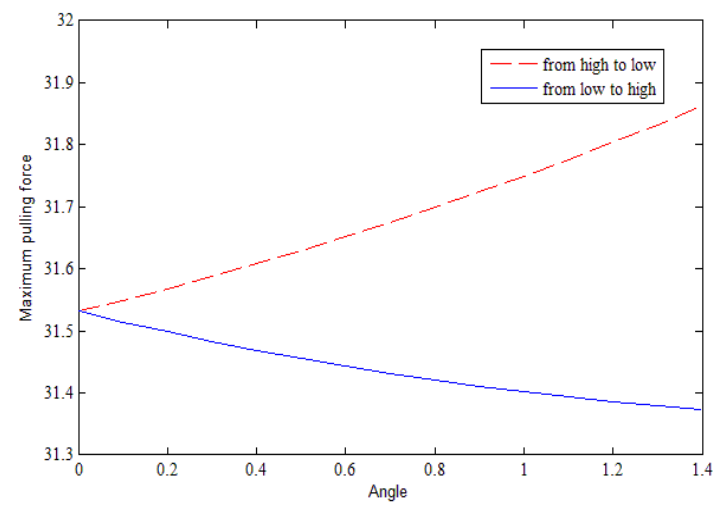

Figure 6. Analysis of position strategy

At last, we analyze the impact of weight difference. From equation (9), we know that a heavier man will produce larger pulling force by assuming that there is no sliding between rope and hands. We take the backward angle is at frictional angle value. Then we simulate the influence of weight. The Figure 7 shows that a $20 \mathrm{~kg}$ gap of weight will produce 160 newtons' gap of pulling force.

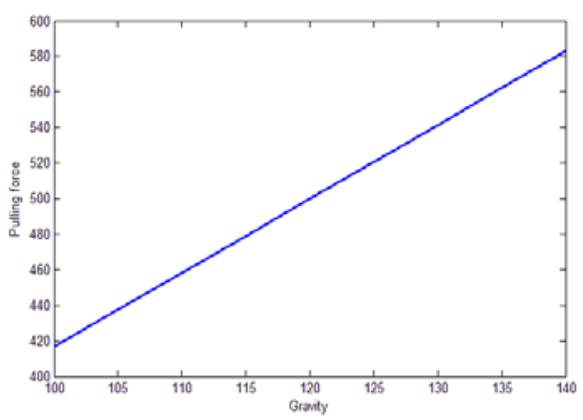

Figure 7. Analysis of the influence of weight

Based on the analysis above, we carry out the position strategies: 
1. Team members stand from high to low. It can produce larger frictional force and maximum pulling force.

2. The heavier you are, the further you stand. The heavier man stand at the end of the team will make the rope tighter, which can decrease the loss of force. And it can also stabilize the rope, and then enhance the pulling efficient.

\section{Evaluation of rules}

A valid and efficient rule should ensure the competitiveness and the fairness of the game. According to Tug of War International Federation (TWIF), the pulling distance for win is 4 meters. If the pulling distance for win is too long, the physical exertion will be too large and the audience will also feel tedious about the game. But if the distance for win is too short, randomness will affect the result of the game, which is unfair for both teams. Thus we need to set an appropriate distance for win.

In an accidental situation, we consider the balance is broken by a surprise extra force. The sliding distance during the occurrence of accident and rebalance is called reaction distance, which should not exceed the distance for win. This procedure can be divided into two stages. In the first stage, team A obtain a surprise extra force which break the force balance. The whole team B will make uniformly accelerated motion. The analysis of stage 1 is as Figure 8.
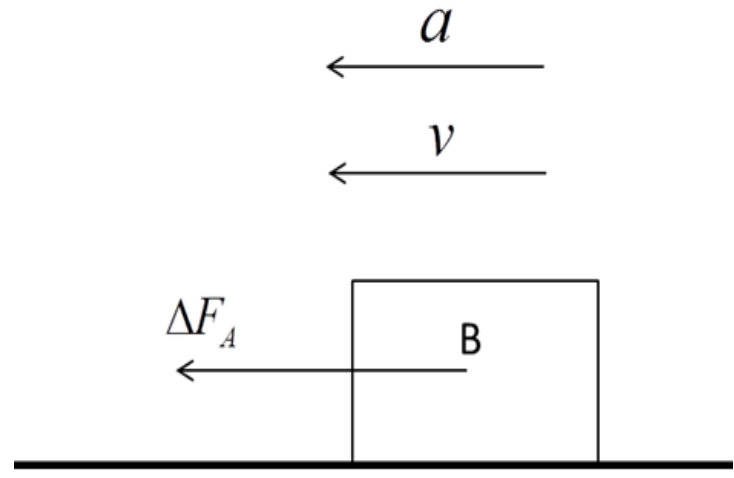

Figure 8. Analysis of the first stage
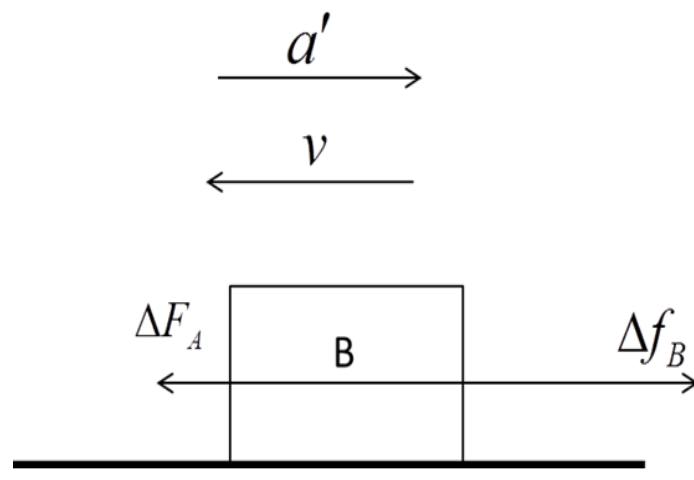

Figure 9. Analysis of the second stage

The acceleration of team $B$ is:

$$
a_{B}=\frac{\Delta F_{A}}{m_{b}}
$$

Then team B adjusts to this accident in case of losing the game. So we have the second stage, see Figure 9. The acceleration of team B changes as:

$$
a_{B}^{\prime}=\frac{\Delta f_{B}-\Delta F_{A}}{m_{b}}
$$

In the first stage, frictional force increase gradually until $\Delta F_{a}=\Delta f_{B}$. This period is very short which is assuming as $t$ second. And because we seem this procedure as a uniformly accelerated motion, the sliding distance can be calculated.

$$
l_{1}=\frac{1}{2} a t^{2}
$$

Then the second stage can also be seemed as a uniformly decelerated motion. The sliding distance can be calculated.

$$
l_{2}=\frac{1}{2} a^{\prime} t^{2}
$$


The total reaction distance is $L=l_{1}+l_{2}$. We assume that the first stage takes 1 second to react and the surprise extra force is 500 newtons. We simulate the situations of $400-\mathrm{kg}, 500-\mathrm{kg}$ and $700-\mathrm{kg}$ class respectively. The result is showed in the picture below.

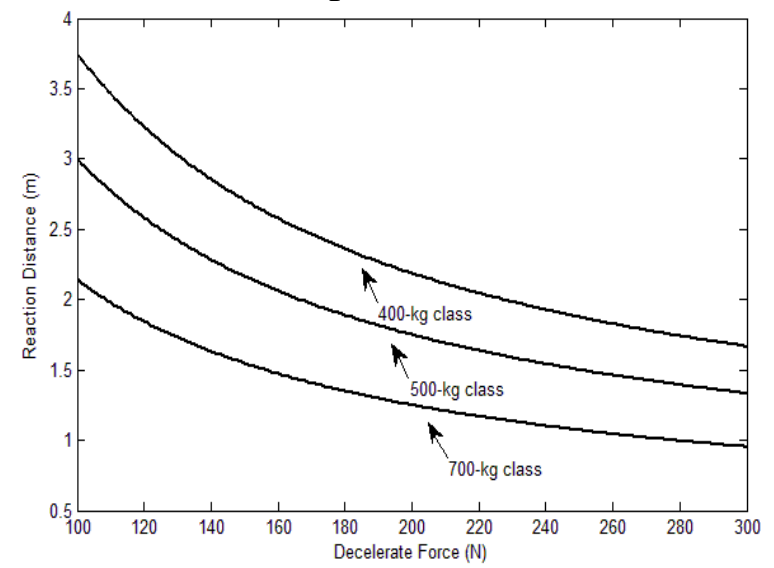

Figure 10. Simulation of reaction distance

As we can see from the simulation result, the reaction distances are all below 4 meters. It indicates that when an accident occurs, team member have time to react to it and the reaction distance will not exceed 4 meters. That prevents an accidental victory and ensures the fairness of the game.

\section{Conclusion}

This paper established a basic framework for tug-of-war and discusses its related position strategies. By simulating different situations, the position strategies were put forward. The two basic position strategies are "standing from high to low" and "the heavier you are, the further you stand". Based on the framework, this paper evaluates the current rules about distance for win. According to the simulation result, we believe the 4-meters to win rule is suitable. The framework and the strategies are scientific, which can provide a valuable reference for athletes and coaches.

\section{References}

[1] Katsue, Ami, Naotoshi, Motoi and Hiroh, Biomechanical analysis on dynamic pulling skill for elite indoor tug of war athletes. Proceedings of 23th International Symposium on Biomechanics in Sports, (2005), pp330-333.

[2] Tu J.H., Lee J.S., Chiu Y.H., The analysis of pulling force curves in tug-of-war. Proceedings of 23th International Symposium on Biomechanics in Sports, (2005), pp487-490.

[3] Masahiro, Fuki, Katsue, Shigeki and Hiroh, Characteristic of pulling movement for Japanese elite tug of war athletes. Proceedings of 23th International Symposium on Biomechanics in Sports, (2005), pp475-478.

[4] Tenenbaum G., Acute and residual effects of vibratory stimulation on explosive force in elite and amateur athletes. Journal of Sports Sciences, No.17, pp.117-182, (1999).

[5] Issurin V.B. Liebermann D.G, Tenenbaum G., Effect of vibratory stimulation training on maximal force and flexibility. Journal of Sports Sciences. 12(6), pp561-566, (1994).

[6] Information on http://www.tugofwar-twif.org/. 